\title{
ESTRATEGIAS PARA LA ASISTENCIA A LA MARCHA DE SUJETOS CON HEMIPARESIA A TRAVÉS DE UNA ÓRTESIS ACTIVA DE RODILLA
}

\author{
Julio S. Lora-Millan ${ }^{1}$, Juan C. Moreno ${ }^{2}$, Eduardo Rocon ${ }^{1}$ \\ ${ }^{1}$ Centro de Automática y Robótica, Consejo Superior de Investigaciones Científicas - Universidad \\ Politécnica de Madrid (CSIC-UPM), Madrid \\ ${ }^{2}$ Grupo de Neuro-Rehabilitación, Consejo Superior de Investigaciones Científicas (CSIC), Madrid. \\ Correspondencia: julio.lora@csic.es
}

\begin{abstract}
Resumen
La hemiparesia es una de las consecuencias motoras más comunes del accidente cerebrovascular. La marcha hemiparética está caracterizada por una fuerte asimetría, y los pacientes que la sufren desarrollan mecanismos de compensación para lograr una marcha funcional; no obstante, estos pacientes siguen requiriendo de dispositivos que asistan su marcha. En este documento proponemos dos estrategias para generar patrones de asistencia que sean implementados por una órtesis activa de rodilla. Ambas estrategias están basadas en la cinemática de la extremidad no afecta del usuario y se coordinan con su movimiento gracias a la estimación en tiempo real de la fase de la marcha. La generación de estos patrones de asistencia ha sido validada con datos experimentales de tres sujetos sanos. Los resultados obtenidos demuestran que la asistencia generada es acorde al movimiento del lado no afecto y está sincronizada con el movimiento del usuario.
\end{abstract}

Palabras clave: Asistencia de la marcha, Hemiparesia, Oscilador de Frecuencia Adaptativo, Órtesis activa de rodilla.

\section{INTRODUCCIÓN}

El accidente cerebrovascular es una de las causas principales de invalidez en la sociedad moderna [1]. En Europa, su incidencia alcanza los 1,1 millones de pacientes al año [2], de los cuales un $80 \%$ desarrolla problemas de movilidad [3]. La hemiparesia es uno de los principales efectos del ictus y conlleva una marcha fuertemente asimétrica debido a la pérdida de movilidad en un lateral del cuerpo [4]. Como consecuencia, los pacientes desarrollan mecanismos de compensación para lograr una marcha funcional [5]. Así mismo, estos pacientes tienden a ralentizar la velocidad de su marcha [6] y les supone un gran consumo metabólico [7].
A pesar de las recientes terapias de rehabilitación, los pacientes no suelen restaurar al completo su movilidad y requieren de dispositivos que asistan su marcha [8]. Recientemente, numerosos exoesqueletos robóticos y órtesis activas tratan de ayudar a estos usuarios en sus actividades de la vida diaria [9].

Sin embargo, aún no se conoce cómo los usuarios responden a la asistencia proporcionada por estas órtesis activas, especialmente cuando esta asistencia se basa en el propio movimiento del usuario. Por otro lado, a la hora de valorar la asistencia prestada por el robot, también se busca poder analizar tanto el efecto directo que tiene sobre la extremidad impedida, como los efectos indirectos que tiene sobre la dinámica global de la marcha.

En el marco del proyecto REFLEX (symmetRy rEinForcer uniLateral powEred eXoskeleton), hemos diseñado una órtesis activa de rodilla para sujetos hemiparéticos para mejorar la simetría de su marcha. Nuestro objetivo es utilizar la propia cinemática del lateral no afecto para generar la asistencia que debe prestar el dispositivo. Para ello se han propuesto dos estrategias de control, la primera replica directamente el movimiento de la pierna sana sobre la impedida, mientras que la otra utiliza el movimiento de la pierna sana para aplicar un patrón sano normalizado sobre la pierna afectada. Trabajos anteriores se han basado en $n$ máquinas de estados finitos para imponer un patrón de marcha sano [10], [11], o han replicado directamente el movimiento de la pierna sana sobre la impedida en basándose en el cálculo del tiempo que se han de retrasar ambos movimientos [12].

Este trabajo utiliza el dispositivo ya presentado en [13] para implementar nuevos controladores que proporcionen asistencia al usuario. La originalidad de esta aportación radica en basar el controlador en la estimación en tiempo real de la fase de la marcha obtenida a través de un Oscilador Adaptativo de Frecuencia (Adaptive Frequency Oscillator - AFO) para generar la asistencia requerida en la pierna afecta en base al movimiento de la pierna no parética, en lugar de las máquinas de estados finitos reportadas 


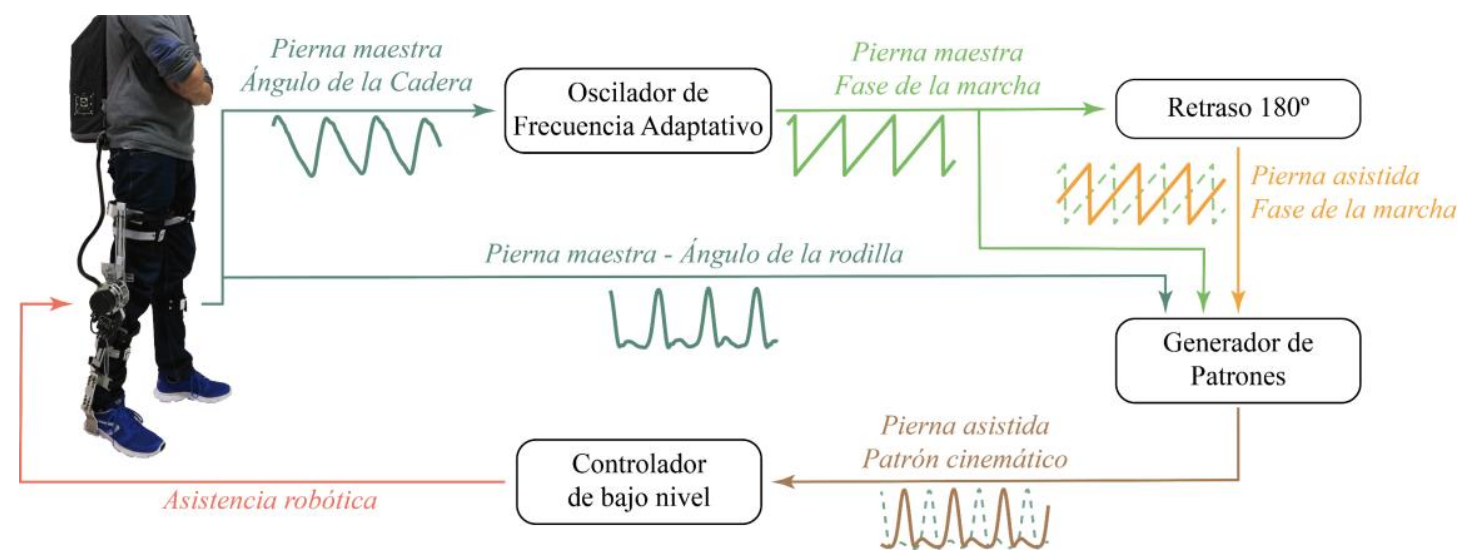

Figura 1: Estrategia de control del dispositivo REFLEX: La asistencia proporcionada por el exoesqueleto robótico se sincroniza con el movimiento de la pierna maestra. El ángulo de la cadera de esta pierna alimenta un oscilador de frecuencia adaptativo (AFO) para estimar en tiempo real la fase de la marcha de la pierna maestra. Esta fase es retrasada $180^{\circ}$ para obtener la fase de marcha para la pierna asistida. La fase de la marcha estimada para ambas piernas y el movimiento de la rodilla de la pierna maestra se utiliza para generar el patrón a seguir por el exoesqueleto de manera que asista el movimiento de la otra extremidad.

previamente. En el presente documento se presentan las estrategias de asistencia siguiendo los dos paradigmas planteados, así como una primera validación de los patrones de asistencia con tres sujetos sanos.

\section{ESTRATEGIAS DE CONTROL PARA LA ASISTENCIA DE LA MARCHA HEMIPARÉTICA}

El objetivo principal de REFLEX es prestar asistencia a la pierna parética de sujetos con hemiparesia de acuerdo con el movimiento de su pierna no afecta (también llamada pierna maestra). De acuerdo con este paradigma, la Figura 1 representa el funcionamiento global del controlador de este dispositivo. Dicho controlador utiliza la información cinemática de la pierna maestra para sincronizar la asistencia que se debe prestar a la pierna parética.

Esta sincronización se basa en la estimación de la fase de la marcha en tiempo real que es calculada por el AFO. Esta herramienta matemática, aprovecha la forma de onda cuasi senoidal del ángulo de flexión de la cadera para estimar la fase de la marcha de la pierna maestra. Dado que los movimientos de ambas piernas están desfasadas $180^{\circ}$ en una marcha sana [14], la fase de la marcha en la pierna asistida se estima retrasando $180^{\circ}$ la fase de marcha calculada en tiempo real por el AFO. Esta fase deseada, junto con la cinemática y la fase de la pierna maestra, son utilizadas por el generador de patrones para calcular la cinemática deseada para la pierna asistida.

\subsection{ESTIMACIÓN DE LA FASE DE LA MARCHA EN TIEMPO REAL.}

Como ya se ha mencionado, REFLEX utiliza un AFO para estimar en tiempo real la fase de la marcha de la pierna maestra. En este contexto, la fase es una variable que aumenta monótonamente en el rango $[0,2 \pi]$ y se restablece cuando se completa el ciclo de la marcha.

Los AFOs son sistemas dinámicos que se pueden sincronizar con señales periódicas aprendiendo sus características como variables de estado [15]. Siendo $\theta_{m}$ el ángulo de flexión de la cadera de la pierna maestra, un AFO puede estimar su fase $\varphi_{m}(t)$ de acuerdo con el siguiente sistema dinámico [16]:

$$
\begin{gathered}
\varepsilon(t)=\theta_{m}(t)-\hat{\theta}_{m}(t) \\
\dot{\omega}=-v_{\omega} \varepsilon(t) \sin \varphi_{m} \\
\dot{\varphi}_{m}=\omega-v_{\varphi} \varepsilon(t) \sin \varphi_{m} \\
\dot{\alpha}_{k}=\eta \cos \left(k \varphi_{m}\right) \varepsilon(t) \quad\left(k=0, \ldots, N_{f}\right) \\
\dot{\beta}_{k}=\eta \sin \left(k \varphi_{m}\right) \varepsilon(t) \quad\left(k=0, \ldots, N_{f}\right) \\
\hat{\theta}_{m}=\sum_{k=0}^{N_{f}} \alpha_{k} \cos \left(k \varphi_{m}\right)+\beta_{k} \sin \left(k \varphi_{m}\right)
\end{gathered}
$$

Donde $\varphi_{m}$ y $\omega$ son la fase y la frecuencia del oscilador sincronizado con la pierna maestra; $\alpha_{k}$ y $\beta_{k}$ son los coeficientes de Fourier utilizados para estimar $\hat{\theta}_{m} \mathrm{y} \varepsilon(t)$ es el error en dicha estimación. $v_{\omega} \mathrm{y} v_{\varphi}$ son constantes de aprendizaje y $\eta$ es un factor de 
acoplamiento que determinan la respuesta dinámica del error $\varepsilon(t)$. En cada instante de ejecución, se considera una nueva entrada $\theta_{m}$ que permite la actualización de todas las variables involucradas.

De cara a otorgar significado fisiológico a la fase estimada por el AFO, es necesario introducir un factor de corrección de manera que el inicio del contacto del pie con el suelo coincida con la fase cero de la marcha $\left(\varphi_{m}=0\right)$. Siguiendo el método propuesto por [17], la fase estimada se corrige mediante un offset $\rho$, el cual es actualizado cada vez que se detecta el evento de inicio de la fase de apoyo.

$$
\begin{aligned}
& \rho=\varphi_{m_{\text {Contacto }}} \\
& \hat{\varphi}_{m}=\varphi_{m}-\rho
\end{aligned}
$$

donde $\hat{\varphi}_{m}$ es la fase de la marcha corregida. Para evitar que la corrección de la fase provoque cambios bruscos en la estimación, el factor de corrección es filtrado mediante un filtro Butterworth paso bajo de primer orden con frecuencia de corte de $0.5 \mathrm{~Hz}$.

Una vez que se ha adquirido la fase de la pierna maestra, esta se retrasa $\pi$ rad para estimar la fase de la pierna asistida $\varphi_{a}$ de acuerdo con la siguiente ecuación:

$$
\varphi_{a}=\bmod \left(\left(\hat{\varphi}_{m}+\pi\right), \pi\right)
$$

Se han realizado los siguientes cambios de variable, de manera que estas pasen a tener significado para el análisis de la marcha.

$$
\begin{aligned}
& \phi_{m}(t)=\frac{\hat{\varphi}_{m}(t)}{2 \pi} \cdot 100 \\
& \phi_{a}(t)=\frac{\varphi_{a}(t)}{2 \pi} \cdot 100
\end{aligned}
$$

$$
f(t)=\frac{\omega(t)}{2 \pi}
$$

De esta manera, las fases de la marcha $\phi_{m}(t)$ y $\phi_{a}(t)$ están en el rango 0-100 y se refieren al porcentaje dentro del paso. Del mismo modo, la frecuencia de la marcha $f(t)$ es la frecuencia en tiempo real en pasos por segundo.

\subsection{ESTRATEGIAS PARA LA ASISTENCIA A LA MARCHA HEMIPARÉTICA}

Una vez que el sistema ha estimado la fase del movimiento de ambas piernas, el algoritmo es capaz de generar un patrón de asistencia según el movimiento medido en la pierna maestra. Para ello, se han desarrollado dos estrategias de asistencia diferentes. La primera de ellas tiene como objetivo replicar el movimiento promedio de la pierna maestra, mientras que la segunda pretende aplicar un patrón sano estándar de manera síncrona con el movimiento del lateral no afecto.

\subsubsection{Estrategia de replicación}

Siguiendo el paradigma introducido por Wang et al. [12], se ha desarrollado una estrategia de asistencia que pretende replicar el movimiento de la pierna maestra del usuario. El concepto de esta estrategia está representado en la Figura 2

En una primera etapa, se considera el movimiento de flexión / extensión de la rodilla de la pierna maestra, el cual se mapea sobre la fase de la marcha estimada para esta pierna. Mediante interpoladores lineales, el sistema reconstruye la cinemática del paso en 50 puntos, separados regularmente un $2 \%$ del paso. Esta información es almacenada en un búfer que contiene la cinemática de los últimos cinco pasos; calculándose el patrón promedio como la media del contenido de este buffer. Este patrón promedio será utilizado como patrón de asistencia en función de la fase estimada para la pierna asistida $\left(\theta_{\text {ref }}\left(\phi_{a}\right)\right)$.

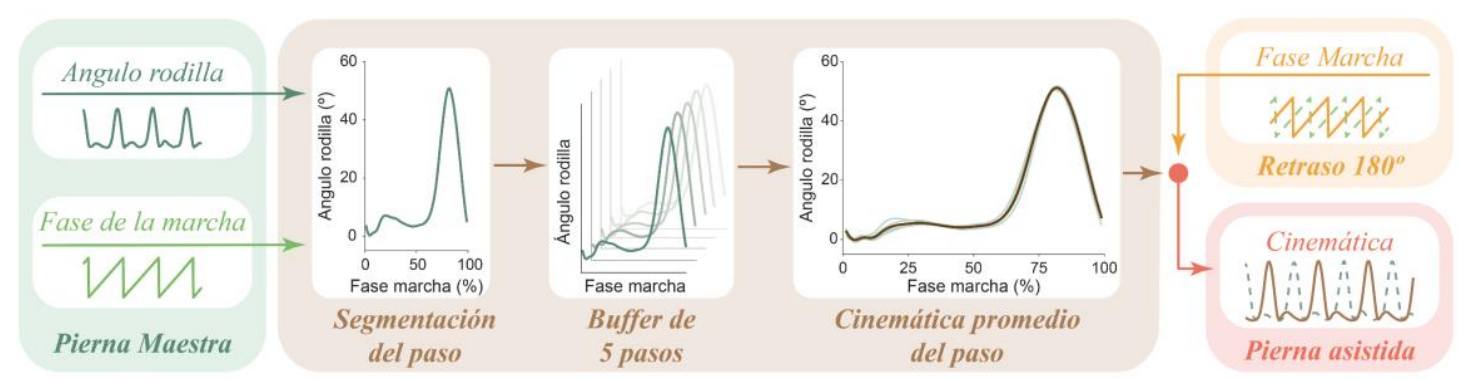

Figura 2: Estrategia de replicación para el control del dispositivo: la cinemática de los últimos cinco pasos se almacena en un buffer para calcular el movimiento promedio en función de la fase de la marcha. Este patrón promedio será aplicado por el exoesqueleto de acuerdo con la fase estimada para la extremidad asistida. 
Para mejorar el control del dispositivo, no sólo se genera el patrón de valores angulares, sino que también las trayectorias de velocidad y aceleración angulares que debe seguir la articulación. Para ello se parte del patrón promedio, suavizado por un filtro Butterworth paso bajo (quinto orden, frecuencia de muestreo de $50 \mathrm{~Hz}$ y frecuencia de corte de $10 \mathrm{~Hz}$ ), el cual es derivado respecto la fase para obtener los patrones de velocidad y aceleración, de acuerdo con las siguientes ecuaciones:

$$
\begin{array}{r}
\dot{\theta}_{r e f}\left(\phi_{a}\right)=\frac{d \theta_{r e f}\left(\phi_{a}\right)}{d t}=\frac{d \theta_{r e f}\left(\phi_{a}\right)}{d \phi_{a}} \cdot \frac{d \phi_{a}}{d t} \\
\ddot{\theta}_{r e f}\left(\phi_{a}\right)=\frac{d^{2} \theta_{r e f}\left(\phi_{a}\right)}{d t^{2}}=\frac{d^{2} \theta_{r e f}\left(\phi_{a}\right)}{d \phi_{a}{ }^{2}} \cdot\left(\frac{d \phi_{a}}{d t}\right)^{2}
\end{array}
$$

Los valores instantáneos de $\theta_{r e f}, \dot{\theta}_{r e f}$ and $\ddot{\theta}_{r e f}$ se calculan interpolando linealmente los puntos que definen los tres patrones cinemáticos teniendo en cuenta la fase estimada para la pierna asistida, así como su derivada.

\subsubsection{Estrategia de sincronización de patrón normalizado}

Esta segunda estrategia se basa en la aplicación de las trayectorias para asistencia robótica de la marcha publicadas por Koopman et al. [18]. Esta estrategia utiliza la información de la pierna maestra para adaptar el patrón estándar al movimiento del usuario, representándose esquemáticamente en la Figura 3.

En una primera etapa, esta estrategia analiza el patrón cinemático de la pierna maestra para extraer el rango de movimiento y la fase en la que se produce la máxima flexión de la rodilla en cada paso. Estas características se almacenan en un búfer de cinco pasos, utilizándose el promedio de los cinco últimos pasos para escalar y desplazar el patrón de referencia. Para evitar cambios bruscos, filtros Butterworth paso bajo de primer orden y frecuencia de corte de $0,5 \mathrm{~Hz}$ suavizan estos factores antes de su aplicación.

Al igual que la estrategia anterior, también se generan referencias en velocidad y aceleración. En este caso, se han calculado previamente las derivadas del patrón angular con respecto a la fase de la marcha para, posteriormente, escalarlas y desplazarlas por los mismos factores que la referencia angular. Mediante la aplicación de las ecuaciones (13) y (14), estas derivadas con respecto a la fase de la marcha se pasan al dominio del tiempo, para utilizarse como referencias de velocidad y aceleración en la pierna asistida. Una vez que estos patrones de referencia están completamente definidos, se utilizan splines cúbicos para interpolar los valores de $\theta_{r e f}, \dot{\theta}_{r e f} \mathrm{y} \ddot{\theta}_{r e f}$ de acuerdo con la fase estimada para el movimiento asistido.

\section{VALIDACIÓN EXPERIMENTAL}

En esta sección, se va a detallar la validación experimental que se ha realizado sobre la generación de las trayectorias de asistencia. Para ello, se reclutaron

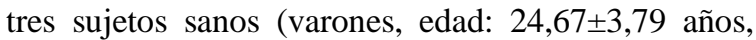
altura: $1,78 \pm 0,02 \mathrm{~m}$, media \pm desviación estándar) cuya marcha fue adquirida para simular la acción de los controladores y validar su funcionamiento. Estos sujetos vistieron sensores inerciales en las extremidades inferiores y sensores de presión en las plantillas para monitorizar la cinemática y eventos de la marcha mientras andaban sobre una cinta rodante. Los sujetos realizaron dos tareas diferentes: (1) caminar durante dos minutos a una velocidad constante que fue seleccionada por ellos mismos para resultar confortable; y (2) caminar a velocidad variable en el rango entre $1 \mathrm{~km} / \mathrm{h}$ y $3 \mathrm{~km} / \mathrm{h}$ (la velocidad se varió aleatoriamente en saltos de $0.2 \mathrm{~km} / \mathrm{h}$ cada $15 \mathrm{~s}$ ). Puesto

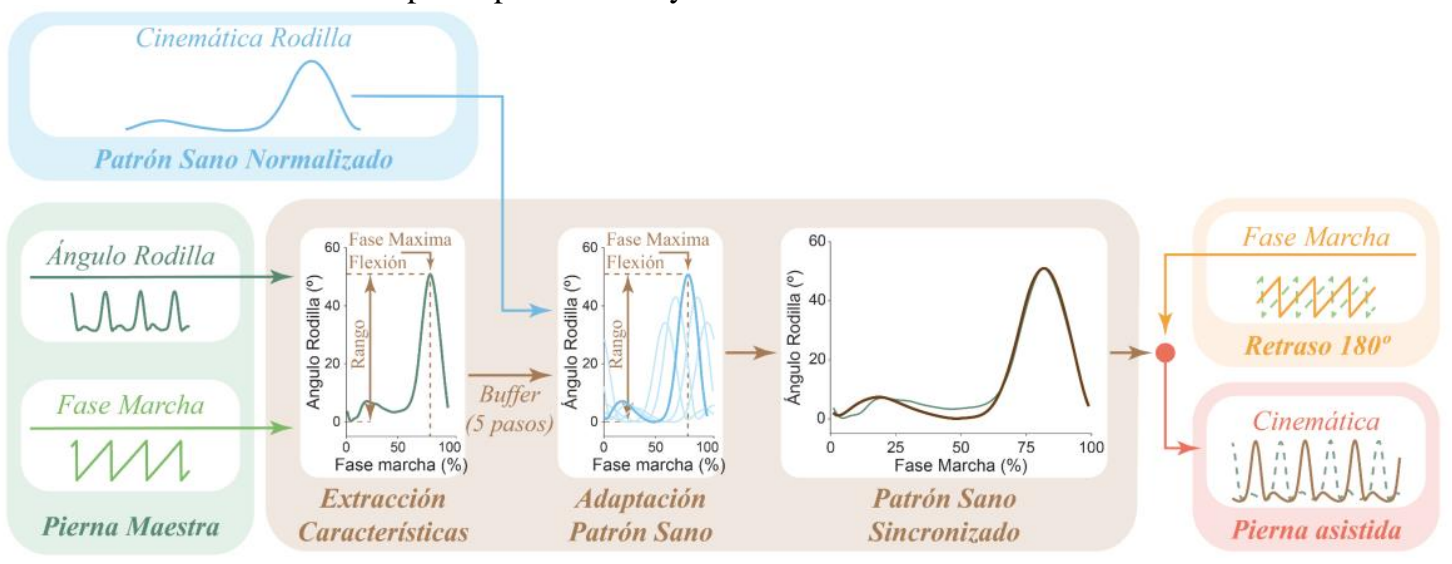

Figura 3: Estrategia de sincronización de un patrón normalizado. Dicho patrón es escalado y desplazado en función de las características de los últimos cinco pasos completados por la extremidad no afecta.

Este nuevo patrón adaptado a la marcha del paciente será aplicado por el exoesqueleto de acuerdo con la fase estimada para la extremidad asistida. 
que se trata de sujetos sanos, el objetivo de la validación es comparar los patrones generados por ambas estrategias con el movimiento de la pierna derecha (pierna equivalente) de los sujetos cuando se utiliza el movimiento de la pierna izquierda (pierna maestra) como base de los algoritmos.

\subsection{ESTIMACIÓN DE LA FASE DE LA MARCHA EN TIEMPO REAL}

Para evaluar el rendimiento del AFO, se utilizaron los datos de los ensayos a velocidad variable; concretamente, utilizamos el ángulo de la cadera izquierda y el contacto del talón izquierdo como entradas del AFO para estimar la fase en tiempo real. Esta fase estimada ha sido comparada con la fase real de la marcha calculada en base a los eventos de contacto del pie, considerando cada inicio del contacto como el comienzo de un nuevo paso.

La Figura 4 representa la fase estimada por el AFO durante un ensayo; nótese cómo el AFO se adapta a los cambios de velocidad manteniendo válidas las estimaciones. El error medio obtenido en la estimación de la fase es de $0.28 \pm 2.36 \%$, con un valor RMS de $2.37 \%$.

\subsection{GENERACIÓN DE LOS PATRONES DE ASISTENCIA}

Para evaluar la generación del patrón de asistencia, se han utilizado los datos experimentales para simular la acción del controlador. Se ha considerado la pierna izquierda como la pierna maestra por lo que el patrón generado debe estar sincronizado con el movimiento de la pierna derecha (pierna equivalente). La Figura 4 muestra el patrón generado por ambas estrategias
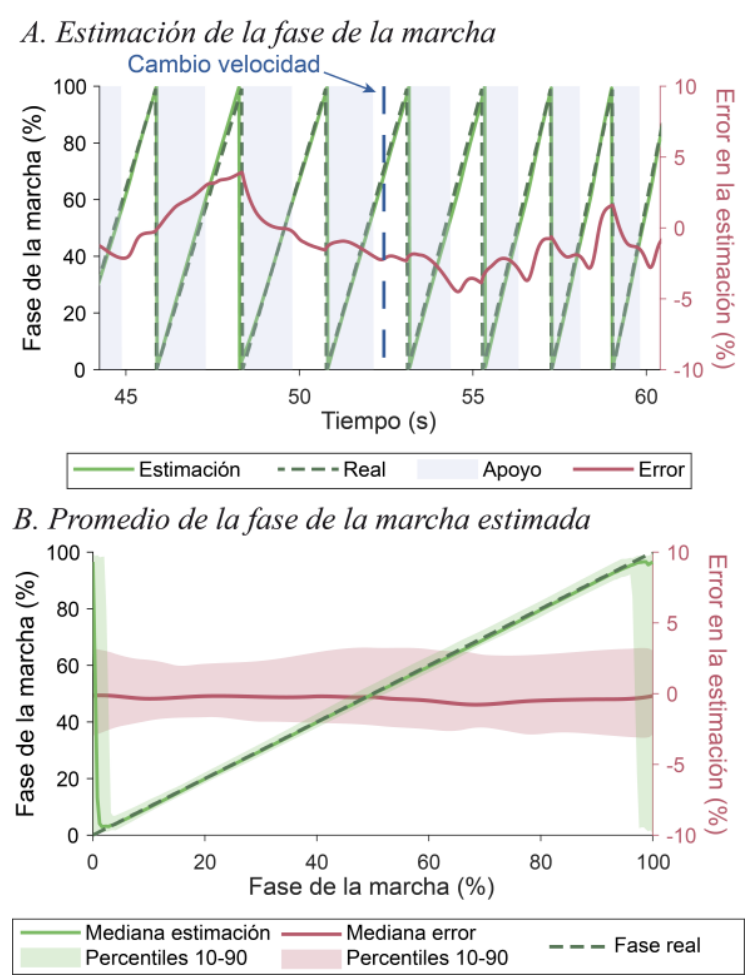

Figura 5: Ejemplo de los resultados de la estimación de la fase de la marcha durante un ensayo a velocidad variable. El panel A compara la fase estimada por el AFO con la fase real durante un intervalo del ensayo. El panel B muestra la mediana y percentiles $10-90$ de la estimación y el error obtenido. En ambos paneles el error se representa respecto el eje derecho.

durante una porción de un ensayo a velocidad variable; nótese cómo el controlador reacciona al cambio en la velocidad de la marcha con una pérdida mínima de sincronía.
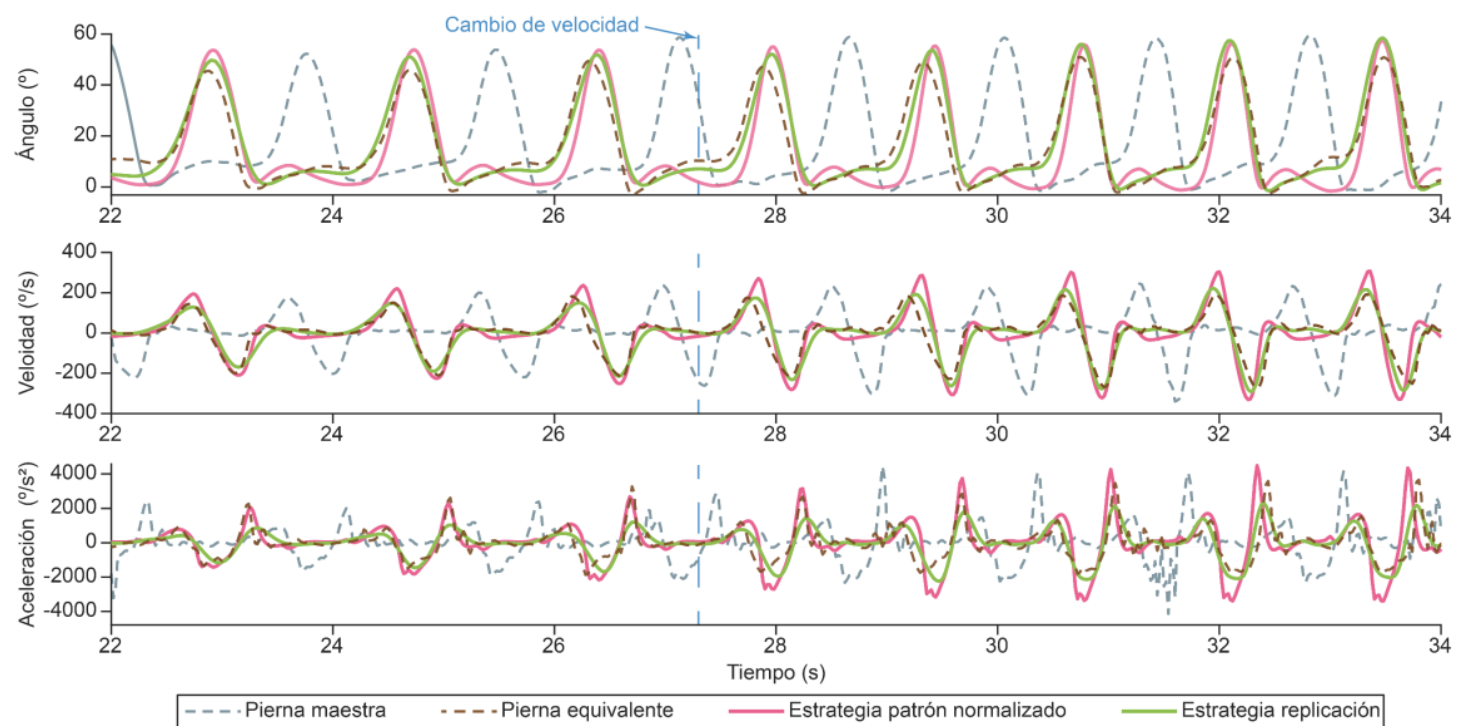

Figura 4: Ejemplo de la generación del patrón de asistencia en base a los datos experimentales de un sujeto sano. Se muestra un ejemplo de las referencias calculadas de acuerdo con las dos estrategias de asistencia y se comparan con el movimiento de la pierna maestra y de la pierna equivalente. 
Con el fin de validar el patrón generado por las estrategias de asistencia, se evalúan tanto la forma de onda del patrón generado como su temporización. Para valorar la forma de onda, se utiliza la representación en phase portraits de los patrones cinemáticos (representación del ángulo de flexión frente a la

A. Velocidad de marcha variable
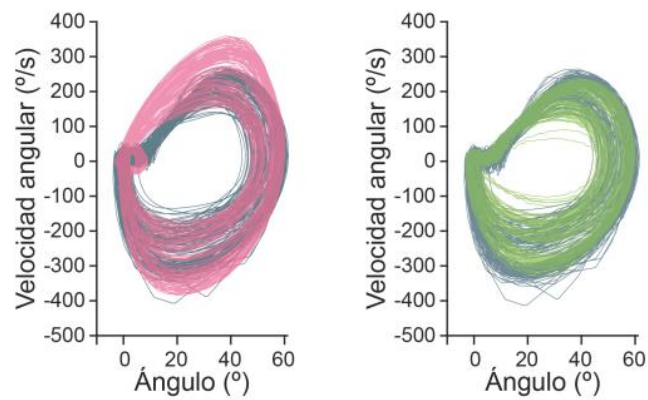

B. Velocidad de marcha constante
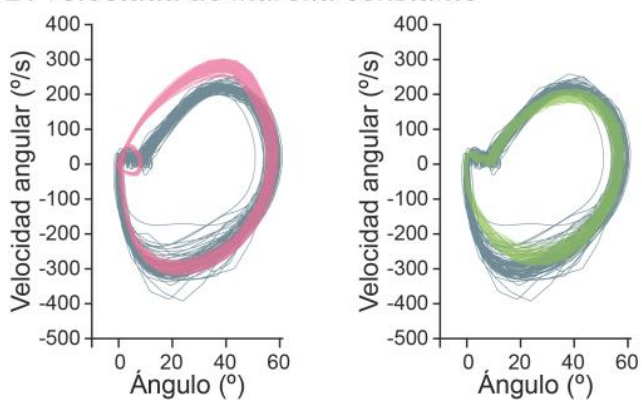

- Pierna maestra

- Estrategia de sincronización de patrón normalizado - Estrategia de replicación

Figura 6: Phase portraits para la comparación del movimiento de la pierna maestra con las

referencias generadas por ambas estrategias. Se muestran los resultados de un sujeto bajo las condiciones de velocidad de marcha variable (panel A) y velocidad constante (panel B) velocidad angular, Figura 6) que describen la dinámica del movimiento[19]. La similitud se evalúa de acuerdo con la siguiente métrica:

$$
\text { Similitud }(\%)=\frac{P 1 \cap P 2}{P 1 \cup P 2} \cdot 100
$$

Donde $P 1$ y $P 2$ son las áreas encerradas por ambos phase portraits, siendo $P 1 \cap P 2$ el área común a ambos y $P 1 \cup P 2$ la unión de ambas áreas. El panel A de la Figura 7 muestra la distribución de esta métrica en función de la estrategia de asistencia y la velocidad de la marcha.

En cuanto a la validación de la temporización del patrón generado, se ha comparado este movimiento con el realizado por la pierna equivalente, es decir, la pierna que no fue utilizada como base de la generación. Esta temporización se ha evaluado a través de dos métricas: la correlación entre ambos movimientos (Figura 7, panel B) y el retraso en la flexión máxima de rodilla (Figura 7, panel C)

La similitud promedio del patrón de asistencia con el movimiento de la pierna maestra es del $77.5 \pm 5.92 \%$ para la estrategia basada en el patrón normalizado y del $84.31 \pm 8.43 \%$ para la estrategia de replicación. En cuanto a la temporización de la asistencia, la correlación promedio es de $\mathrm{R}=0.9 \pm 0.1$ y el retardo promedio es de $-0.021 \pm 0.060$ s para la estrategia basada en el patrón normalizado mientras que la estrategia de replicación obtuvo una correlación de $\mathrm{R}=0.95 \pm 0.08$ y un retraso promedio de $-0.012 \pm 0.053 \mathrm{~s}$.

Después de comprobar la no normalidad de los datos en algunas de las distribuciones (test de KolmogorovSmirnov, $\mathrm{P}<0.01)$ se han buscado diferencias significativas entre estrategias así como el efecto de
A. Similitud

Referencia vs Pierna maestra

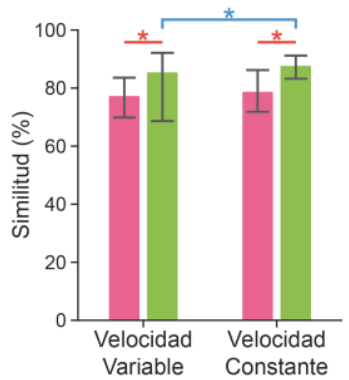

B. Correlación

Referencia vs Pierna equivalente

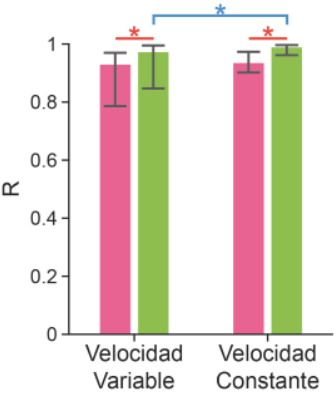

C. Máximo retraso en flexión Referencia vs Pierna equivalente

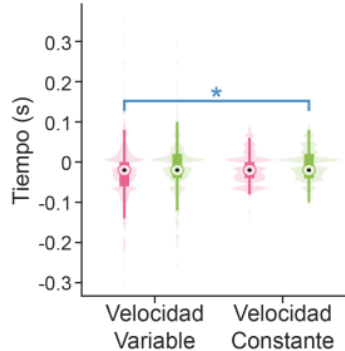

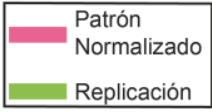
Constante

Figura 7: Evaluación de la referencia generada por las dos estrategias de asistencia. El panel A muestra la similitud del phase portrait entre la referencia y el movimiento de la pierna maestra. El panel B muestra la correlación entre la referencia y el movimiento de la pierna equivalente. La longitud de las barras indica la mediana de la métrica y los bigotes los percentiles 10-90. El panel C muestra el retardo en la flexión máxima de rodilla entre la referencia y la pierna equivalente; la distribución está representada por su violin plot (boxplot junto al histograma en forma de área). Los marcadores (*) indican diferencias significativas entre las distribuciones 
variaciones en la velocidad de la marcha; están representadas por los marcadores de la Figura 7 (test de Kruskall-Wallis, $\mathrm{P}<0.01$ ).

\section{$4 \quad$ DISCUSIÓN}

En este documento se presentan dos estrategias para generar patrones de referencia para asistencia unilateral de la marcha en pacientes hemiparéticos. Ambas estrategias se basan en la estimación en tiempo real de la fase de la marcha mediante un AFO y en la medición de la cinemática de la pierna maestra, es decir, la extremidad sana, del paciente. Ambas estrategias generan un patrón de asistencia que está sincronizado con el propio movimiento del paciente, una de ellas mediante la replicación del movimiento de la pierna no afecta y la otra mediante la sincronización de un patrón sano estándar que se adapta al movimiento del usuario.

La estimación en tiempo real de la fase de la marcha ha demostrado ser capaz de adaptarse a los cambios de velocidad de la marcha, manteniendo un error RMS inferior a $2.4 \%$. Esto está en línea con los resultados publicados por otros trabajos que también utilizan un AFO para la estimación en tiempo real de la fase de marcha: Zheng et al. obtuvieron errores RMS del 3\% mediante el uso de sensores capacitivos [20] y Yan et al. reportaron errores RMS del $2 \%$ en base a medidas de la fuerza de reacción del suelo, y del $1.4 \%$ en base al ángulo de la cadera medida a través de un encoder [21].

En cuanto a la generación de los patrones de asistencia, cabe destacar que la estrategia de replicación obtiene mejores métricas que la estrategia basada en el patrón normalizado, reportando similitudes promedio con la pierna maestra sobre el $84 \%$ y el $77 \%$ y correlaciones del 0.95 y 0.9 respectivamente. Estas discrepancias son debidas a la diferencia existente entre el patrón normalizado y el propio patrón del sujeto, siendo, lógicamente, más similar la asistencia a su propio patrón cuando se utiliza la estrategia de replicación

Por otra parte, si bien la estrategia basada en el patrón normalizado no muestra cambios ante variaciones de velocidad de la marcha, la estrategia de replicación obtiene mejores resultados a velocidad constante. Esto es debido a que, el cálculo del patrón promedio es más consistente cuando el sujeto camina a velocidad constante pues supone una mejor estimación de la fase de la marcha por parte del AFO y el propio patrón de la pierna sana es más repetitivo si no existen cambios de velocidad.

Comparadas con las estrategias de control reportadas previamente para el prototipo REFLEX, esta metodología se basa en la estimación de la fase de la marcha realizada por el AFO, la cual es una señal continua y robusta ante cambios de velocidad. Frente a las estrategias basadas en retrasos y máquinas de estados finitos [13] esta nueva metodología permite un cálculo más robusto del patrón de asistencia y permite cerrar el lazo de control, no sólo en posición sino también en velocidad y aceleración para un mejor seguimiento de trayectorias.

\section{CONCLUSIÓN}

En este documento se han introducido dos nuevas estrategias de control para una órtesis activa de rodilla que tienen por objeto asistir la marcha de sujetos hemiparéticos en base al movimiento de su pierna no afecta. Las estrategias presentadas han sido evaluadas en base a datos experimentales obtenidos de sujetos sanos y han sido validadas tanto desde el punto de vista de la dinámica del movimiento y como de la sincronía con la propia marcha de los sujetos.

Las estrategias presentadas en este trabajo permitirán la asistencia de la marcha de sujetos con hemiparesia y permitirán llevar a cabo nuevas terapias de rehabilitación que busquen aumentar la simetría de la marcha y la reducción de mecanismos de compensación. Así mismo, se abre la posibilidad a realizar nuevos estudios para analizar el efecto que tiene la asistencia robótica sobre estos pacientes y estudiar cómo el movimiento de los propios pacientes se adapta a la asistencia prestada por el dispositivo.

\section{Agradecimientos}

Este trabajo ha sido financiado por los Programas de Actividades I+D en la Comunidad de Madrid y los Fondos Estructurales de la Unión Europea (Robocity, S2018/NMT-4331). El autor Julio S. Lora Millán ha recibido financiación del programa de Formación de Profesorado Universitario del Ministerio de Universidades del Gobierno de España (ref. FPU16/01313).

\section{English summary}

\section{STRATEGIES FOR HEMIPARETIC GAIT ASSISTANCE TRHOUGH AN ACTIVE KNEE ORTHESIS}

\begin{abstract}
Hemiparesis in one of the most common consequences of stroke. Hemiparetic gait is characterized by a strong asymmetry and patients usually develop compensation mechanism to achieve a functional gait; however, these patients still need devices that assist their gait. In this document, we propose two strategies
\end{abstract}


to generate assistive gait patterns for an active knee orthosis. Both strategies are based on the kinematics of the unimpaired limb and use a real-time gait phase estimation to synchronize the generated pattern with the movement of the user. We have validated these pattern generation with experimental data from three healthy subjects. Results point out that generated assistance is in accordance with the motion of the sound limb and it is synchronized with the movement of the user.

Keywords: Gait assistance, Hemiparesis, Adaptive Frequency Oscillator, Active Knee Orthosis.

\section{Referencias}

[1] G. Morone et al., "Robot-assisted gait training for stroke patients: Current state of the art and perspectives of robotics," Neuropsychiatr. Dis. Treat., vol. 13, pp. 1303-1311, 2017.

[2] Y. Béjot, H. Bailly, J. Durier, and M. Giroud, "Epidemiology of stroke in Europe and trends for the 21st century," Press. Medicale, vol. 45, no. 12, pp. e391-e398, 2016.

[3] S. Li, G. E. Francisco, and P. Zhou, "Post-stroke hemiplegic gait: New perspective and insights," Front. Physiol., vol. 9, no. AUG, pp. 1-8, 2018.

[4] S. J. Olney and C. Richards, "Hemiparetic gait following stroke. Part I: Characteristics," Gait Posture, vol. 4, no. 2, pp. 136-148, Apr. 1996.

[5] S. Schmid, K. Schweizer, J. Romkes, S. Lorenzetti, and R. Brunner, "Secondary gait deviations in patients with and without neurological involvement: A systematic review," Gait Posture, vol. 37, no. 4, pp. 480-493, 2013.

[6] N. E. Mayo et al., "Disablement following stroke," Disabil. Rehabil., vol. 21, no. 5-6, pp. 258-268, Jan. 1999.

[7] D. J. Farris, A. Hampton, M. D. Lewek, and G. S. Sawicki, "Revisiting the mechanics and energetics of walking in individuals with chronic hemiparesis following stroke: From individual limbs to lower limb joints," J. Neuroeng. Rehabil., vol. 12, no. 1, pp. 1-12, 2015.

[8] L. E. Skolarus, J. F. Burke, and V. A. Freedman, "The role of accommodations in poststroke disability management.," J. Gerontol. B. Psychol. Sci. Soc. Sci., vol. 69 Suppl 1, no. Suppl 1, pp. S26-34, Nov. 2014.

[9] M. F. Bruni, C. Melegari, M. C. De Cola, A. Bramanti, P. Bramanti, and R. S. Calabrò, "What does best evidence tell us about robotic gait rehabilitation in stroke patients: A systematic review and meta-analysis," J. Clin. Neurosci., vol. 48, pp. 11-17, Feb. 2018.

[10] L. N. Awad et al., "A soft robotic exosuit improves walking in patients after stroke," Sci. Transl. Med., vol. 9, no. 400, p. eaai9084, Jul. 2017.

[11] W.-Y. Lai, H. Ma, W.-H. Liao, D. T.-P. Fong, and K.-M. Chan, "HIP-KNEE control for gait assistance with Powered Knee Orthosis," in 2013
IEEE International Conference on Robotics and Biomimetics (ROBIO), 2013, pp. 762-767.

[12] W. J. Wang, J. Li, W. Da Li, and L. N. Sun, “An Echo-Based Gait Phase Determination Method of Lower Limb Prosthesis," Adv. Mater. Res., vol. 706-708, pp. 629-634, Jun. 2013.

[13] J. S. Lora-Millan, J. C. Moreno, and E. Rocon, "Assessment of gait symmetry, torque interaction and muscular response due to the unilateral assistance provided by an active knee orthosis in healthy subjects," in 2020 8th IEEE RAS/EMBS International Conference for Biomedical Robotics and Biomechatronics (BioRob), 2020, vol. 2020Novem, no. 688175, pp. 229-234.

[14] M. Plotnik, N. Giladi, and J. M. Hausdorff, “A new measure for quantifying the bilateral coordination of human gait: Effects of aging and Parkinson's disease," Exp. Brain Res., vol. 181, no. 4, pp. 561-570, 2007.

[15] R. Ronsse, S. M. M. De Rossi, N. Vitiello, T. Lenzi, M. C. Carrozza, and A. J. Ijspeert, "RealTime Estimate of Velocity and Acceleration of Quasi-Periodic Signals Using Adaptive Oscillators," IEEE Trans. Robot., vol. 29, no. 3, pp. 783-791, Jun. 2013.

[16] G. Aguirre-Ollinger, A. Narayan, and H. Yu, "Phase-Synchronized Assistive Torque Control for the Correction of Kinematic Anomalies in the Gait Cycle," IEEE Trans. Neural Syst. Rehabil. Eng., vol. 27, no. 11, pp. 2305-2314, Nov. 2019.

[17] W. van Dijk, C. Meijneke, and H. van der Kooij, "Evaluation of the Achilles Ankle Exoskeleton," IEEE Trans. Neural Syst. Rehabil. Eng., vol. 25, no. 2, pp. 151-160, Feb. 2017.

[18] B. Koopman, E. H. F. van Asseldonk, and H. van der Kooij, "Speed-dependent reference joint trajectory generation for robotic gait support," $J$. Biomech., vol. 47, no. 6, pp. 1447-1458, Apr. 2014.

[19] Y. Hurmuzlu, C. Basdogan, and J. J. Carollo, "Presenting joint kinematics of human locomotion using phase plane portraits and Poincaré maps," $J$. Biomech., vol. 27, no. 12, pp. 1495-1499, Dec. 1994.

[20] E. Zheng, S. Manca, T. Yan, A. Parri, N. Vitiello, and Q. Wang, "Gait phase estimation based on noncontact capacitive sensing and adaptive oscillators," IEEE Trans. Biomed. Eng., vol. 64, no. 10, pp. 2419-2430, 2017.

[21] T. Yan, A. Parri, V. Ruiz Garate, M. Cempini, R. Ronsse, and N. Vitiello, "An oscillator-based smooth real-time estimate of gait phase for wearable robotics," Auton. Robots, vol. 41, no. 3, pp. 759-774, 2017.

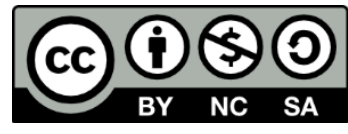
(C) 2021 by the authors. Submitted for possible open access publication under the terms and conditions of the Creative Commons Attribution CC BY-NC-SA 4.0 license (https://creativecommons.org/licenses/byncsa/4.0/deed.es). 